Moulines, C. Ulises

\title{
Cuatro tipos de desarrollo teórico en las ciencias empíricas
}

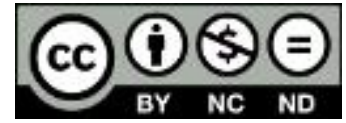

Esta obra está bajo una Licencia Creative Commons Argentina.

Atribución - No Comercial - Sin Obra Derivada 2.5

https://creativecommons.org/licenses/by-nc-nd/2.5/ar/

Documento descargado de RIDAA-UNQ Repositorio Institucional Digital de Acceso Abierto de la Universidad Nacional de Quilmes de la Universidad Nacional de Quilmes

\section{Cita recomendada:}

Moulines, C. U. (2011). Cuatro tipos de desarrollo teórico en las ciencias empíricas. Metatheoria, 1(2), 11-27. Disponible en RIDAA-UNQ Repositorio Institucional Digital de Acceso Abierto de la Universidad Nacional de Quilmes http://ridaa.unq.edu.ar/handle/20.500.11807/2551 


\title{
Cuatro tipos de desarrollo teórico en las ciencias empíricas"
}

Four Types of Theoretical Development in Empirical Sciences

\author{
C. Ulises Moulines
}

\begin{abstract}
Resumen
En este artículo se arguye que pueden distinguirse cuatro tipos fundamentales de estructuras diacrónicas en la ciencia y que estos cuatro tipos pueden representarse formalmente mediante una versión refinada del aparato del estructuralismo metateórico. Los cuatro tipos pueden describirse como: cristalización, evolución teórica, incrustación y suplantación con inconmensurabilidad (parcial). Ellos se elucidan primeramente en términos intuitivos, informales, y se sugieren algunos ejemplos históricos (algunos de los cuales ya se han reconstruido en la literatura estructuralista). En la segunda parte del ensayo, los cuatro tipos se caracterizan formalmente en términos estructuralistas; en ello tienen un papel central las nociones de subestructura parcial escalonada y de red teórica diacrónica. Se exponen los ejemplos del desarrollo de la termodinámica fenomenológica de Clausius como caso de cristalización, y de la mecánica newtoniana y de la termodinámica gibbsiana como casos de evolución teórica.
\end{abstract}

Palabras clave: cristalización - evolución teórica - incrustación - inconmensurabilidad - redes teóricas - conjunto-escalón - subestructura parcial

\begin{abstract}
This paper argues that four fundamental types of diachronic structures in science may be distinguished, and that these four types can be represented formally through a refined version of the apparatus of metatheoretical structuralism. These four types may be described as: crystallization, theory-evolution, embedding, and replacement with (partial) incommensurability. They are first explicated in intuitive, informal terms, and some historical examples (some of which have already been reconstructed in the structuralist literature) are suggested for each type. In the second part of the essay, the four types are formally characterized in structuralistic terms; the notions of a partial substructure as an echelon-set and of a diachronic theorynet thereby play a central role. The examples of the development of Clausius's phenomenological thermodynamics as an instance of a crystallization process, and of Newtonian mechanics and Gibbsian thermodynamics as instances of theory-evolutions are laid out.
\end{abstract}

Keywords: crystallization - theory-evolution - embedding - incommensurability - theory-nets echelon-sets - partial substructures

* Recibido: 16 Enero 2011. Aceptado en versión revisada: 9 Marzo 2011.

† Seminar für Philosophie, Logik und Wissenschaftstheorie, University of Munich. Para contactar al autor, por favor escriba a: moulines@lrz.uni-muenchen.de.

* Debo a dos revisores anónimos de una primera versión de este ensayo algunas propuestas de mejoras que se han incluido en la versión final. Este trabajo participa del proyecto de investigación PICTR 2006 № 2007, de la Agencia Nacional de Promoción Científica y Tecnológica, de la República Argentina.

Metatheoria 1(2)(2011): 11-27. ISSN 1853-2322.

(C) Editorial de la Universidad Nacional de Tres de Febrero. Publicado en la República Argentina. 


\section{Introducción}

El objetivo general de este ensayo es presentar una tipología estructuralista del desarrollo teórico de las ciencias empíricas que reúna las siguientes condiciones: a) ser intuitivamente (históricamente) plausible; b) ser formalmente precisa; c) ser uniforme y sistemática, es decir, proponer un esquema general que sirva para encajar en él las diversas formas diacrónicas de la ciencia; d) ser aplicable a la reconstrucción de casos concretos ("el movimiento se demuestra andando").

Desde sus inicios, la metateoría estructuralista se propuso mostrar su pertinencia no solo para un análisis diferenciado de las diversas estructuras sincrónicas de las teorías, sino también para la elucidación de sus aspectos diacrónicos. Ya en el último capítulo de The Logical Structure of Mathematical Physics (1971), Joseph D. Sneed hizo algunas insinuaciones en ese sentido; de manera más extensa y sistemática, la segunda parte de Theorienstrukturen und Theoriendynamik (1973) de Wofgang Stegmüller está dedicada a la reconstrucción estructuralista (avant la lettre) de las estructuras diacrónicas de la ciencia, en especial de la ciencia normal y la "ciencia revolucionaria" de Thomas Kuhn, así como de los "programas de investigación" de Imre Lakatos. Más adelante, Wolfgang Balzer, Joseph Sneed y yo mismo dedicamos el Capítulo V de nuestra Architectonic for Science (1987), a desarrollar un aparato formal adecuado para tratar por lo menos una forma diacrónica determinada de la ciencia, lo que allí llamamos "evoluciones teóricas"; ella corresponde más o menos a la "ciencia normal" de Kuhn y a los "programas de investigación" de Lakatos. Y aplicamos dicho aparato a la reconstrucción de dos casos reales de la historia de la ciencia: la evolución de la mecánica newtoniana y la de la termodinámica gibbsiana. En años subsiguientes, he vuelto una y otra vez a considerar las posibilidades de aplicación del estructuralismo al análisis diacrónico de las teorías científicas (véase Moulines 1982, 1991 y 1996). Sin embargo, es en el presente ensayo donde propongo por primera vez un esquema formal generalizado para el tratamiento uniforme de todas las formas de desarrollo teórico en las ciencias. Además, propongo una elucidación formal de un tipo de desarrollo que hasta ahora ha recibido poca atención tanto dentro del estructuralismo como fuera de él: lo que llamo "cristalizaciones teóricas". Esta elucidación está basada en mi análisis reciente de un caso concreto, el desarrollo de la termodinámica de Clausius (véase Moulines 2010).

\section{El trasfondo historicista}

Aparte de los estructuralistas, los únicos filósofos de la ciencia que han intentado de manera sistemática construir esquemas generales para analizar la diacronía de las teorías han sido, como es bien sabido, los autores llamados historicistas y, en particular, Kuhn, Lakatos y Larry Laudan (véase Kuhn 1962, Lakatos 1970, Laudan 1977). Pero también es bien sabido que los esquemas propuestos por estos autores brillan por su vaguedad e incontrolabilidad ante ejemplos concretos. Al parecer, ellos eran de la opinión de que es impracticable aplicar herramientas 
formales o cuasi formales a cuestiones de la filosofía diacrónica de la ciencia. ${ }^{1}$ El resultado de esta actitud metodológica también es consabido: los filósofos interesados por la diacronía de la ciencia se han vuelto rabiosamente antiformalistas y los filósofos de la ciencia que aún creen en la utilidad de los instrumentos formales se han desinteresado por la diacronía de la ciencia. Los estructuralistas nos hemos esforzado por navegar entre esa Scylla y esa Caribdis y creo que lo hemos hecho con bastante éxito. Aquí propongo iniciar una nueva etapa en ese difícil camino.

\section{Diacronía tetravalente de la ciencia}

Incluso dejando de lado las insuficiencias de las metateorías diacrónicas de Kuhn, Lakatos y Laudan debidas a su vaguedad y falta de control (meta-)empírico, un defecto del que adolecen es que parten de un esquema demasiado simplificado para detectar las diversas formas de desarrollo teórico. En efecto, podemos decir que es común a esos autores que su esquema sea solo bivalente. Es decir, distinguen solo dos formas diacrónicas: en Kuhn, ellas son la "ciencia normal" y la "ciencia revolucionaria"; en Lakatos son el desarrollo teórico dentro de un programa de investigación y la competencia entre programas de investigación rivales; en Laudan, se trata, o bien del desarrollo de una tradición de investigación, o bien de la tensión entre tradiciones de investigación distintas. Mi tesis es, por el contrario, que (independientemente de cualquier metateoría concreta como pueda ser el estructuralismo) necesitamos partir de un esquema que sea por lo menos tetravalente por lo que respecta a los tipos de desarrollo teórico. Es decir, pueden detectarse por lo menos cuatro tipos fundamentales distintos de desarrollo. Los podemos denominar así:

1) emergencia o cristalización de teorías;

2) evolución de teorías;

3) incorporación o incrustación de una teoría en otra;

4) suplantación de una teoría por otra acompañada de inconmensurabilidad parcial.

Por supuesto, como en cualquier otra ciencia de la cultura (y la filosofía de la ciencia, por su objeto, forma parte de las ciencias de la cultura), se trata de tipos ideales en el sentido de Max Weber. Pero aunque sean "ideales" son también lo bastante concretos y sustanciales como para que con ellos podamos ordenar el material histórico adecuadamente. Habrá, sin duda, casos históricos concretos de difícil clasificación, o casos intermedios; pero ello no le quita a la tipología su importancia y utilidad. ${ }^{2}$

\footnotetext{
${ }^{1}$ Para ser justos con Kuhn, él admitió en alguna ocasión la posibilidad de que ciertas herramientas formales, en especial las del estructuralismo, pudieran contribuir de manera significativa a la filosofía diacrónica de la ciencia (véanse sus observaciones en Kuhn 2000). Sin embargo, él mismo nunca se adentró por ese camino.

${ }^{2}$ No afirmo que la lista de los cuatro tipos mencionados sea exhaustiva, aunque tampoco estoy seguro de que no lo sea. José Luis Falguera me hizo notar, en un comentario a una versión previa de este texto (presentada
} 
La tarea que se nos plantea a los estructuralistas, en consecuencia, consiste en tratar de elucidar con la mayor precisión posible, empleando las herramientas de nuestra metateoría, cada uno de los tipos (y establecer una comparación entre ellos). Esta tarea ha sido ya realizada de manera bastante completa para el tipo 2) (la evolución de teorías) (véase Moulines 1982, y Balzer, Moulines \& Sneed 1987, Cap. V). Se han dado también ya algunos pasos, aunque insuficientes, para la elucidación de 3) y 4) (véase, entre otros, Balzer, Moulines \& Sneed 1987, Caps. VI y VII, así como Lorenzano 2008 y Caamaño 2009); con respecto a 1) no se ha hecho casi nada. Mi objetivo en este ensayo es elucidar cada uno de los tipos mencionados y hacerlo, además, dentro de un esquema uniforme. Son sobre todo dos nociones formales las que nos permitirán este tratamiento uniforme de la diacronía científica: la noción ya bien conocida de red teórica y la noción (introducida aquí por primera vez) de subestructura parcial escalonada.

Pero antes de pasar a la reconstrucción estructuralista de los cuatro tipos fundamentales de desarrollo teórico mencionados, conviene describirlos de una manera intuitiva, que nos guíe en la construcción de las elucidaciones formales posteriores. Para facilitar la comprensión y proporcionar a la vez una base ulterior de control de las elucidaciones formales respectivas, también daré una lista de ejemplos históricos "paradigmáticos" de cada tipo. Respecto a cada uno de los tipos, mencionaré dos grupos de casos paradigmáticos: aquellos que son plausibles intuitivamente como ejemplos del tipo en cuestión, pero que aún no han sido reconstruidos en la literatura estructuralista, y aquellos que ya han sido reconstruidos estructuralísticamente.

\subsection{Emergencia o cristalización}

En la fase inicial de una disciplina (emergencia) o después de la quiebra de una teoría anterior en una disciplina ya constituida, y a través de un proceso largo y gradual, al que genéricamente podemos denominar "cristalización”, los modelos de una nueva teoría se van construyendo paso a paso, a través de muchos estadios intermedios, fragmentarios, antes de que aparezca una nueva red teórica plenamente desarrollada. Este proceso revela poseer las siguientes características (en terminología estructuralista):

1) Puede haber (aunque ello no es necesario) diversas redes teóricas en competencia durante el mismo periodo, que comparten algunos, pero no todos los componentes de los modelos.

\footnotetext{
en el VII Encuentro Iberoamericano de Metateoría Estructuralista) que es plausible concebir otros tipos de desarrollo científico, además de los señalados. Falguera propone tres más: a) desarrollo "preparadigmático"; b) crisis de una teoría sin suplantación; c) escisión. Ahora bien, el caso a) de Falguera me parece estructuralmente (aunque no históricamente) análogo a lo que llamo "cristalización"; respecto al tipo b), mi apuesta es que los ejemplos históricos correspondientes pueden reconstruirse, ya sea como casos de evolución teórica, o bien como incorporaciones en mi sentido (como el propio Falguera insinúa). Solo el tipo c) parece a primera vista un tipo no reducible a los demás pero, para dirimir esta cuestión, habría que analizar algún ejemplo histórico claro para filtrar a partir de él su estructura general. Sea como sea, prima facie no parecen plantearse obstáculos insuperables para reconstruir esas supuestas escisiones mediante el aparato formal que se expone más abajo.
} 
2) Los elementos teóricos básicos en periodos sucesivos de un proceso de cristalización son esencialmente distintos; es decir, los modelos potenciales, los parciales y los actuales, difieren de una red a otra, aunque comparten algunos componentes.

3) Aunque los elementos teóricos básicos de las redes sucesivas son distintos, algunos elementos no-básicos, es decir, algunas especializaciones, son iguales o cuasi iguales, especialmente en el sentido de que comparten las mismas aplicaciones intencionales, o casi las mismas, y algunos de los conceptos T-teóricos.

4) El proceso de cristalización concluye con el establecimiento de una red teórica arbórea, es decir, con un solo elemento teórico básico, firmemente establecido.

Ejemplos plausibles no reconstruidos: la emergencia de la mecánica aristotélica (s. IVIII a.C.); la emergencia de la astronomía "ptolemaica" (s. III a.C.-s. II d.C.); la cristalización de la mecánica cartesiana después de la quiebra de la dinámica aristotélica (1630-1670); la emergencia de la genética "mendeliana" (1865-1905), o sea, desde los trabajos originales de Mendel hasta el establecimiento del primer "paradigma" genético por parte de Bateson (véase Lorenzano 2006).

Ejemplo reconstruido (parcialmente): la cristalización de la termodinámica fenomenológica después de la quiebra de la teoría del calórico (1825-1878) y, en particular, la cristalización parcial de la termodinámica fenomenológica en los escritos de Rudolf Clausius "Über die bewegende Kraft der Wärme" (1850) y "Über eine veränderte Form des zweiten Hauptsatzes der mechanischen Wärmetheorie” (1854); este último, en realidad, consta de dos ensayos distintos, probablemente concebidos en periodos diferentes (véase Moulines 2010).

\subsection{Evolución teórica}

En este caso, una red teórica se transforma a lo largo de varios periodos añadiendo o suprimiendo algunos elementos teóricos especializados, pero sin perder su identidad esencial, definida por un núcleo teórico básico $K_{0}$ (que consiste siempre del mismo marco conceptual general y la(s) misma(s) ley(es) fundamental(es)). En cada periodo sucesivo aparecen nuevas leyes especiales (= nuevas especializaciones) que son especialización de alguna especialización del periodo precedente. Los conjuntos de aplicaciones intencionales en periodos sucesivos, ni son idénticos, ni están necesariamente en la relación conjuntista de inclusión; pero al menos sus intersecciones no son vacías. Lo que aquí llamamos "evoluciones teóricas" corresponde aproximadamente a la "ciencia normal" de Kuhn, a un "programa de investigación" de Lakatos o a (al menos algunos ejemplos de) una "tradición de investigación" en el sentido de Laudan.

Ejemplos plausibles: la astronomía ptolemaica desde el s. II d.C. hasta mediados del siglo XVI; la química del flogisto a lo largo del siglo XVIII; la teoría del calórico entre 1790 y 1825; la teoría de la relatividad generalizada desde 1916; la genética "mendeliana" (en su segunda fase) desde los trabajos de Morgan y cola- 
boradores a partir de 1910 hasta el advenimiento de la genética molecular en los 1960 s.

Ejemplos reconstruidos: la evolución de la mecánica newtoniana desde 1687 (año de la aparición de los Principia de Newton) hasta principios del siglo XIX; la evolución de la termodinámica fenomenológica desde 1878 (año de la publicación de la segunda parte de la monografía de Gibbs, "On the Equilibrium of Heterogeneous Substances") hasta años cuarenta (véase Balzer, Moulines \& Sneed 1987, Cap. V).

\subsection{Incorporación o incrustación}

Aquí, los modelos (potenciales y actuales) de una teoría previa se incorporan o incrustan (aproximadamente y quizás no completamente) en los modelos de una nueva teoría, más compleja, de modo que todas (o casi todas) las aplicaciones intencionales de la primera teoría, hayan sido exitosas o no, pasan a ser aplicaciones intencionales exitosas de la segunda. Este tipo de proceso responde (presumiblemente) a uno de los sentidos del concepto de "revolución científica" en Kuhn.

Ejemplos plausibles no reconstruidos: la incorporación de la óptica ondulatoria en la electrodinámica de Maxwell y la de esta última en la teoría especial de la relatividad; la incorporación de esta última en la teoría generalizada de la relatividad; la incorporación de la mecánica cuántica (versión Schrödinger o Heisenberg) en la electrodinámica cuántica (de Dirac), y la de esta última en el "modelo estándar" de la física de partículas; la incorporación de la teoría darwiniana de la evolución en la teoría "sintética"; la incorporación de la genética "mendeliana" (en realidad, "morganiana") en la genética molecular.

Ejemplos reconstruidos: la incorporación de la mecánica cartesiana del choque en la mecánica newtoniana (véase Balzer, Moulines \& Sneed 1987, Cap. VI); la incorporación de la teoría planetaria de Kepler en la mecánica newtoniana (véase Balzer, Moulines \& Sneed 1987, Cap. VII).

\subsection{Suplantación con inconmensurabilidad (parcial)}

Una teoría destinada a sistematizar un conjunto dado de aplicaciones intencionales puede ser completamente suplantada (abruptamente o en un plazo relativamente breve) por otra teoría (con un marco conceptual muy diferente) destinada a más o menos el mismo conjunto de aplicaciones. En tal caso, la suplantación es debida a que algunas aplicaciones intencionales de la primera teoría no son exitosas (son "anomalías"), mientras que sí lo son en la nueva teoría. Esto corresponde al sentido más plausible de las "revoluciones científicas" de Kuhn como "cambios de paradigma”. A pesar de la suplantación (y diga lo que diga Kuhn), la nueva teoría recupera algunos de los componentes de los modelos potenciales de la primera y sobre todo muchas de sus aplicaciones intencionales, aunque sea aproximadamente.

Ejemplos plausibles no reconstruidos: la suplantación de la astronomía ptolemaica por la astronomía copernicana; la suplantación de la mecánica aristotélica por la cartesiana; la suplantación de la teoría lamarckiana de la evolución por 
la darwiniana; la suplantación de la mecánica newtoniana por la relativista (caso muy controvertido).

Ejemplo reconstruido: la suplantación de la teoría del flogisto por la teoría del oxígeno de Lavoisier (véase Caamaño 2009).

\section{Conceptos metateóricos generales}

Antes de pasar a la elucidación formal de cada uno de los tipos fundamentales de diacronía científica, conviene introducir algunas nociones metateóricas generales que son necesarias para nuestra tarea y que intervienen en todos los tipos a reconstruir. Las dos nociones clave que operan en todos los casos son las de red teórica diacrónica y la de subestructura parcial escalonada. ${ }^{3}$ Presupongo que el lector ya está familiarizado con las demás nociones estructuralistas estándar, en especial la de red teórica (sincrónica). La noción de subestructura parcial escalonada es de reciente cuño y, por lo que sé, no ha sido definida previamente en la literatura, estructuralista o no-estructuralista. La noción de red teórica diacrónica fue introducida ya en Balzer, Moulines \& Sneed (1987), Cap. V, pero allí fue definida solamente para elucidar el tipo de proceso diacrónico que hemos llamado "evolución teórica”. Aquí se modificará levemente aquella definición para hacerla adecuada a cualquiera de los cuatro tipos de proceso diacrónico.

Para simplificar la exposición, de entre las nociones usuales del estructuralismo prescindiremos en lo sucesivo de las condiciones de ligadura (constraints), de los vínculos ínterteóricos (links) y de los conjuntos borrosos (blurs). Nada esencial se pierde con ello con respecto a la argumentación de este ensayo. Su incorporación a las elucidaciones aquí propuestas sería relativamente fácil aunque tediosa.

\subsection{Subestructuras parciales escalonadas}

Sea $S=\left\langle A_{1}, \ldots, A_{n}\right\rangle$ una estructura con $m$ dominios $D_{1}, \ldots, D_{m}$, y $n-m$ relaciones (con $n>m) R_{m+1}, \ldots, R_{n}^{n}$. Sea $S^{\prime}$ una estructura. Sea $A$ un dominio o una relación.

Def. D.1.1: A $\hat{\in} S$ syss $\exists i\left(1 \leq i \leq n \wedge A=A_{i}\right)$.

La fórmula "A $\hat{\epsilon} S$ " se lee: "A es un componente de $S$ ".

Def. D.1.2: $S^{\prime} \hat{\epsilon} S$ syss $\forall$ A $\hat{\epsilon} S^{\prime}: A \hat{\epsilon} S$.

La fórmula " $S$ ' $\hat{\in} S$ " se lee " $S^{\prime}$ es una subestructura parcial de $S$ ".

Nota: Cualquier componente de una estructura $S$ puede ser considerado trivialmente como una subestructura parcial de $S$.

\footnotetext{
${ }^{3}$ La noción de subestructura (o subsistema) es estándar en la teoría de modelos: una estructura (o sistema) $S$ es subestructura (o subsistema) de otra(o) $S^{*}$ cuando el (los) dominio(s) de $S$ es (son) subconjunto(s) propio(s) o impropio(s) del (de los) dominio(s) de $S^{*}$, y por tanto las relaciones de $S$ son restricciones de las relaciones de $S^{*}$ (véase Manzano 1989); la noción de subestructura parcial está menos divulgada: $S$ es subestructura parcial de $S^{*}$ cuando, además de ser subestructura de $S^{*}$, ocurre que hay por lo menos una relación $R^{*}$ en $S^{*}$ que carece de contrapartida en $S$, es decir, no hay ninguna relación $R$ en $S$ que sea restricción de $R^{*}$. Finalmente, la noción de subestructura parcial escalonada se ha introducido específicamente para el tema que nos ocupa (véase la definición D.1.5 más abajo).
} 
Def. D.1.3: $\Theta$ es la operación conjuntista que consiste en aplicar sucesivamente un número finito de veces las operaciones "Pot" (conjunto-potencia) y " $x$ " (producto cartesiano) a cierto(s) conjunto(s) previamente dado(s), empezando siempre por Pot.

Def. D.1.4: $A$ es un conjunto-escalón sobre $B_{1}, \ldots, B_{m}$ syss $A \in \Theta\left(B_{1}, \ldots, B_{m}\right){ }^{4}$

Ejemplos:

- A es un conjunto-escalón sobre sí mismo, pues $A \in \operatorname{Pot}(A)$, y por tanto $A$ $\in \Theta(A)$.

- Si $A \subseteq B$, entonces $A$ es un conjunto-escalón sobre $B$, pues $A \in \operatorname{Pot}(B)$, y por tanto $A \in \Theta(B)$.

- Si $A \subseteq B \times C$, entonces $A \in \Theta(B, C)$.

- Si $A \subseteq B \times \operatorname{Pot}(C) \times D$, entonces $A \in \Theta(B, C, D)$.

- Etc.

Def. D.1.5: $S$ es una subestructura parcial escalonada de $S^{*}\left(S \eta S^{*}\right)$ syss $\forall S_{i}\left(S_{i} \hat{\in} S\right)$ $\exists S_{k}^{*}\left(S_{k}^{*} \hat{\in} S^{*}\right): S_{i} \in \Theta\left(S_{k}^{*}\right)$.

Teorema: $\eta$ es una relación reflexiva y transitiva, y por tanto induce un cuasi orden en el conjunto de las estructuras definidas en un contexto dado. (La prueba de este teorema es inmediata dada la definición.)

\section{Ejemplos:}

Sean las estructuras $S=\left\langle A_{1}, \ldots, A_{n}\right\rangle$ y $S^{*}=\left\langle B_{1}, \ldots, B_{m}\right\rangle$. Supongamos que $A_{1} \subseteq B_{1}$, y que $A_{2} \subseteq B_{1} \times \operatorname{Pot}\left(B_{3}\right)$. Entonces, $A_{1}, A_{2} y\left\langle A_{1}, A_{2}\right\rangle$ son diversas subestructuras parciales escalonadas de $S^{*}$.

Nota bene: Si $S \eta S^{*}$, entonces la $n$-ariedad de $S$ no tiene por qué ser menor que la $n$-ariedad de $S^{*}$, es decir, puede ocurrir que, si $n$ es la $n$-ariedad de $S$ y $m$ la de $S^{*}$, se dé $n>m$. (Posible ejemplo: la relación entre la estructura espaciotemporal newtoniana y la estructura espaciotemporal relativista; en efecto, sea $E$ el espacio euclidiano tridimensional de la mecánica newtoniana, $T$ la variedad unidimensional que representa el tiempo en la mecánica newtoniana, y $V$ la variedad tetradimensional que representa el espacio-tiempo en la mecánica relativista. Entonces $\langle E, T\rangle \eta V$, pues $E \in \Theta(V)$ y $T \in \Theta(V)$.)

\subsection{Redes teóricas diacrónicas}

Def. D.2.1: $T$ es un elemento teórico diacrónico syss existen $K, I, C C, h, G$ tales que
(A) $T=\langle K, I, G\rangle$
(B) $\langle K, I\rangle$ es un elemento teórico
(C) CC es una comunidad científica
(D) $h$ es un periodo histórico
(E) $G$ es una generación de científicos y $\exists g: g(h, C C)=G$
(F) G tiene la intención de aplicar $K$ a I

\footnotetext{
${ }^{4}$ La noción de conjunto-escalón (ensemble échelon en francés, echelon set en inglés) se debe originalmente al grupo Bourbaki en su reconstrucción conjuntista de las matemáticas. Posteriormente esa noción fue implementada para la filosofía de la física por Günther Ludwig y Erhard Scheibe (véase Ludwig 1978, Scheibe 1997).
} 
Def. D.2.2: $N$ es una red teórica diacrónica syss $N$ es un conjunto de elementos teóricos diacrónicos y $\mathbf{T}_{\mathbf{b}}$ es un conjunto de elementos teóricos diacrónicos (llamados "básicos") tales que:
(1) $\mathbf{T}_{\mathbf{b}} \subseteq N$
(2) $\forall T_{i} \in N \exists T_{0} \in \mathbf{T}_{\mathbf{b}}\left(T_{i} \sigma T_{0}\right)$
(3) $\forall T_{i}, T_{j} \in N\left(\mathrm{G}_{i}=\mathrm{G}_{j}\right)$

Def. D.2.3: Sean $T, T^{\prime}$ dos elementos teóricos diacrónicos.

$T^{\prime}$ es una especialización diacrónica de $T\left(T^{\prime} \delta T\right)$ syss
(A) $K^{\prime} \sigma K$
(B) $I^{\prime} \cap I \neq \varnothing$
(C) $h<h^{\prime}$

Nota bene: En este concepto generalizado de especialización diacrónica, no se exige que la comunidad científica $C C$ siga siendo la misma en el tránsito de $T$ a $T^{\prime}$. Esto solo es plausible exigirlo en un determinado tipo de proceso diacrónico, el de las llamadas evoluciones teóricas, pero no en los demás.

Def. D.2.4: Sean $N, N^{\prime}$ dos redes teóricas diacrónicas.

$N^{\prime}$ es posterior a $N\left(N^{\prime} \triangleleft N\right)$ syss

$\exists T^{\prime} \in N^{\prime} \exists T \in N\left(T^{\prime} \delta T\right)$

Def. D.2.5: Sean $N, N^{\prime}$ dos redes teóricas diacrónicas.

$N^{\prime}$ es inmediatamente posterior a $N\left(N^{\prime} \pi N\right)$ syss
(A) $N^{\prime} \triangleleft N$
(B) $\neg \exists N^{*}\left(N \neq N^{*} \neq N^{\prime} \wedge N^{\prime} \triangleleft N^{*} \triangleleft N\right)$

\section{Elucidación formal de los cuatro tipos de procesos diacrónicos}

\subsection{Cristalización teórica}

Sean $n$ redes teóricas $N_{1}, \ldots, N_{n}$. Sea $\mathbf{T}_{\mathbf{i}}^{\mathbf{b}}$ el conjunto de los elementos teóricos básicos de cada $N_{i}\left(\operatorname{con}\left\|\mathbf{T}_{\mathbf{i}}^{\mathbf{b}}\right\| \geq 1\right)$.

$\left\langle N_{1}, \ldots, N_{n}\right\rangle$ es una cristalización teórica syss

(1) Para todo $i<n: N_{i+1} \pi N_{i}$

(2) $\forall i, k\left(1 \leq i, k \leq n \wedge i \neq k \rightarrow \mathbf{T}_{\mathbf{i}}^{\mathbf{b}} \neq \mathbf{T}_{\mathbf{k}}^{\mathbf{b}}\right)$

(3) $\forall i(1 \leq I \leq n): \exists k \exists k^{*}\left(T_{k} \hat{\in} N_{i} \wedge T_{k} \notin \mathbf{T}_{\mathbf{k}}^{\mathbf{b}} \wedge T_{k^{*}} \hat{\in} N_{i+1}\right.$ :

(a) $\forall x \in M_{k} \exists x^{*} \in M_{k^{*}}\left(x \eta x^{*}\right) \wedge$

(b) $\left.\exists S, y, y^{*}\left(y \in I_{k} \wedge y^{*} \in \mathrm{I}_{\mathrm{k}^{*}} \wedge S \eta y \wedge S \eta y^{*}\right)\right)$

De acuerdo con esta definición, lo especifico de una cristalización se resume en tres características: en primer lugar, las redes sucesivas no son necesariamente arbóreas ni tienen necesariamente los mismos elementos teóricos básicos (aunque

\footnotetext{
${ }^{5}$ El símbolo “<” para la relación de precedencia histórica entre periodos debe interpretarse como una relación cualitativa y no cuantitativa. Ello significa, entre otras cosas, que dos periodos distintos que están en la relación "<" pueden solaparse cronológicamente (pueden compartir años o meses, por ejemplo).
} 
pueden compartir algunos de ellos); en segundo lugar, existen al menos algunos elementos teóricos no-básicos de una red precedente cuyos modelos son subestructuras parciales escalonadas (incluyendo posiblemente la identidad) de algún modelo de algún elemento teórico (básico o no) de la red inmediatamente posterior; y, en tercer lugar hay algunas aplicaciones intencionales de una red y de la red que le sigue inmediatamente, las cuales, aunque posiblemente no sean idénticas (por no compartir todos los conceptos T-no-teóricos), comparten al menos una subestructura parcial escalonada (es decir, algunos de los conceptos T-no-teóricos).

El ejemplo de la cristalización de la termodinámica de Clausius cumple con esta caracterización, como puede comprobarse en la sucesión de grafos expuestos a continuación. Nótese en este ejemplo que los elementos teóricos básicos de las tres redes sucesivas son distintos, pero que hay algunas especializaciones, como

Clausius 1a fase (1850)

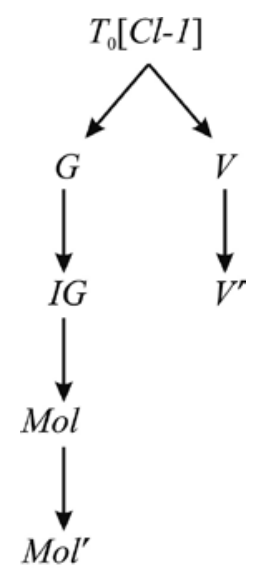

Clausius 2a fase (1854a)

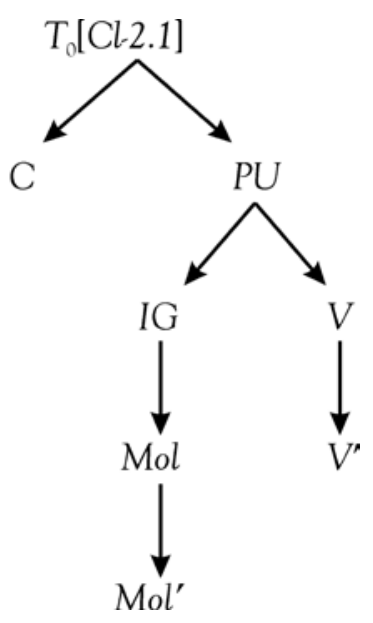


Clausius 3a fase (1854b)

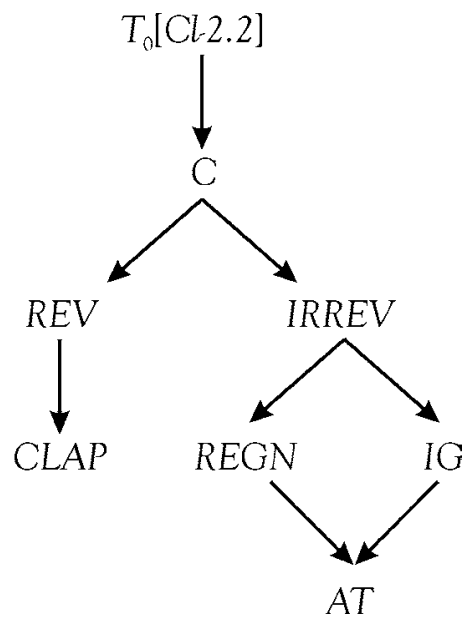

IG (gases ideales) que son comunes a redes sucesivas. El lector interesado en el significado de los símbolos que aparecen en cada nódulo de las redes puede acudir a Moulines (2010). Se trata de las denominaciones de diversos tipos de sistemas termodinámicos estudiados por Clausius.

\subsection{Evolución teórica}

$\left\langle N_{1}, \ldots, N_{n}\right\rangle$ es una evolución teórica syss

(1) $\forall i(1<i \leq n): N_{i} \pi N_{i-1}$

(2) $\exists T_{0} \forall i, k(1 \leq i, k \leq n): \mathbf{T}_{\mathbf{i}}^{\mathbf{b}}=\mathbf{T}_{\mathbf{k}}^{\mathbf{b}}=\left\{T_{0}\right\}$

(3) $\forall N_{i}(1<i \leq n) \forall T_{j} \in N_{i} \exists N_{k}(k<i) \exists T_{h} \in N_{k}\left(T_{j} \delta T_{h}\right)$

(4) $\forall i, k(1 \leq i, k \leq n): C C_{i}=C C_{k}$

Las tres características específicas de una evolución teórica son, por consiguiente, que en ella hay un solo elemento teórico básico $T_{0}$ que rige toda la evolución, que todo elemento teórico de una red anterior es especializado por algún elemento teórico de una red posterior y que la comunidad científica permanece invariable.

En Balzer, Moulines \& Sneed (1987, Cap. V), aparecen reconstruidos con detalle dos ejemplos históricos que son instancias de este concepto de evolución teórica: la evolución de la mecánica newtoniana desde Newton hasta la época de Coulomb y Laplace, así como la evolución de la termodinámica gibbsiana desde Gibbs hasta la época de Nernst y Bridgman. A modo de recordatorio se reproducen aquí los grafos respectivos, con ligeras simplificaciones.

I. La evolución de la mecánica newtoniana (1687-180..):

$N_{1}:(1687-172 .$. 


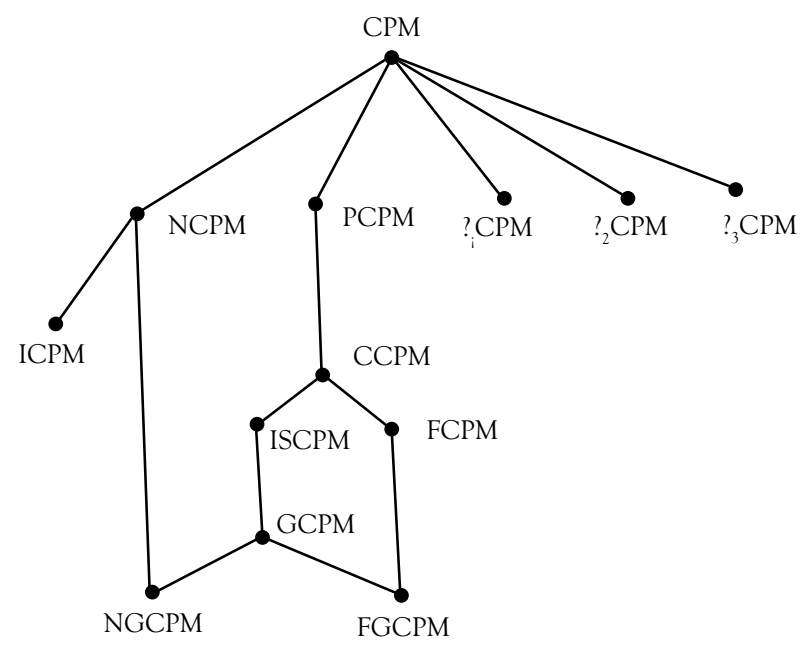

$N_{2}:(172 . .-177 .$.

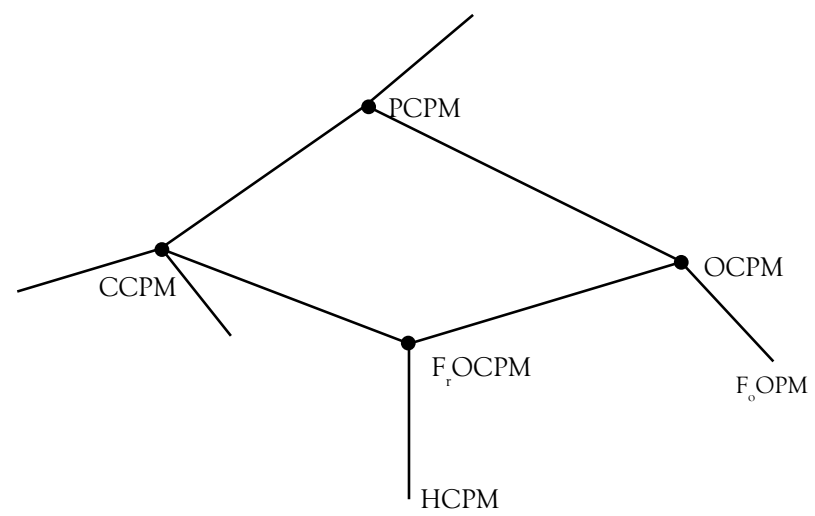

$N_{3}:(177 . .-180 .$.

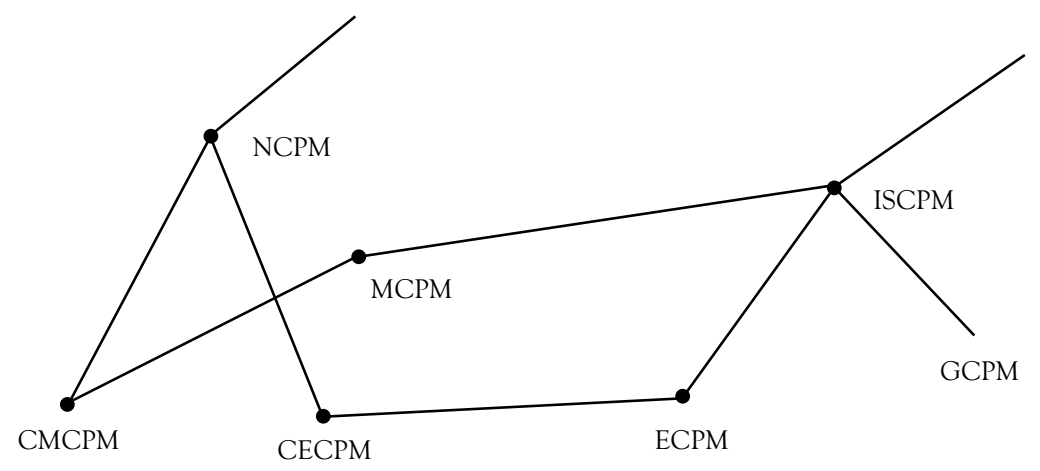


II. La evolución de la termodinámica fenomenológica (1878-194..) $N_{1}:(1878-188 .$.

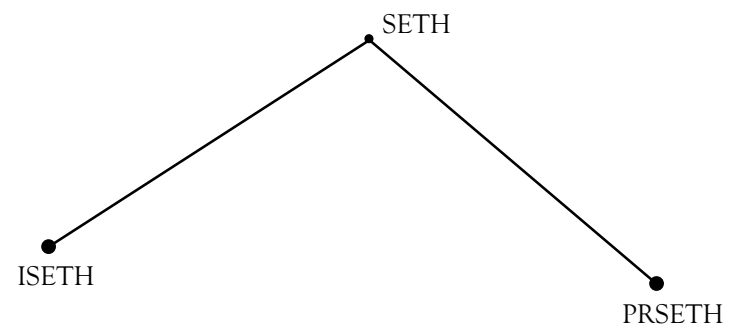

$N_{2}:(188 . .-1906)$

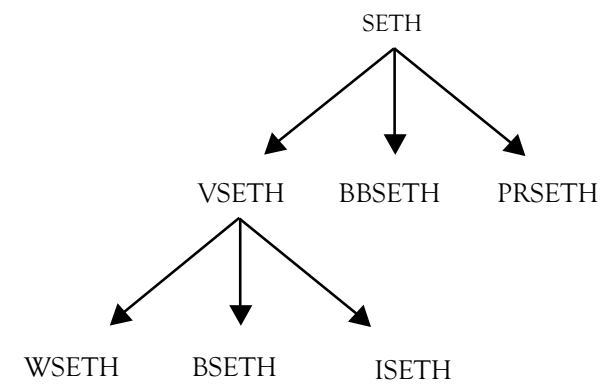

$N_{3}:(1906-194 .$.

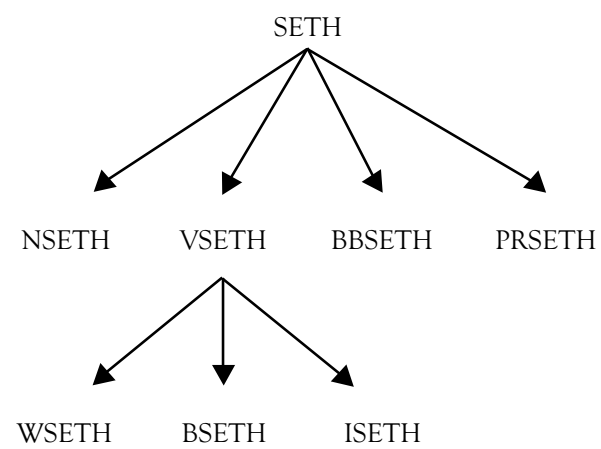




\subsection{Incorporación teórica}

Para definir esta noción formalmente necesitamos previamente la noción auxiliar de "éxito".

Def. E.3.1.: Sea $m$ un modelo actual de un elemento teórico $T_{i}$ de la red $N$. Diremos que $m$ es un "modelo exitoso" en $N$ syss existe una aplicación intencional y de ese elemento teórico, es decir $y \in I_{i}$, tal que $y=r(m)$. Diremos que una aplicación intencional $y \in I_{i}$ es una aplicación exitosa syss existe un modelo actual $m$ de $T_{i}$ tal que $y=r(m)$.

Def. E.3.2.: La red $N$ es incorporable en la red $N^{*}$ syss

(1) Todo modelo exitoso en $N$ es una subestructura parcial escalonada (aproximativa) de algún modelo exitoso en $N^{*}$

(2) Toda aplicación intencional exitosa en $N$ es una subestructura parcial escalonada (aproximativa) de alguna aplicación intencional exitosa en $N^{*}$.

Nota bene: Obsérvese que, en esta comprensión generalizada de la noción de incorporación, en primer lugar no se exige que $N^{*}$ sea estrictamente más exitosa que $N$ : considérese el caso de dos redes empíricamente equivalentes (ejemplos: mecánica newtoniana de partículas y mecánica lagrangiana; mecánica ondulatoria de Schrödinger y mecánica de matrices de Heisenberg). En segundo lugar, tampoco se exige que $N$ preceda diacrónicamente a $N^{*}$ : por razones de mayor simplicidad, didáctica o práctica, puede que posteriormente se construya una red teórica $N^{*}$ que sea incorporable a una $N$ precedente (ejemplo: la mecánica del sólido rígido formulada por Euler medio siglo después de la formulación por Newton de la mecánica de partículas).

Teorema: Las relaciones ínterteóricas de equivalencia, reducción y aproximación son subtipos especiales de incorporación. ${ }^{7}$

\section{Tránsito conmensurable}

Intuitivamente, los fenómenos diacrónicos de evolución e incorporación muestran cierto "aire de familia": en ambos casos se trata de manifestaciones claras de un "progreso científico", en el que los estadios posteriores en el desarrollo aportan más éxitos e informaciones que los estadios precedentes. Por ello puede ser conveniente subsumirlos bajo un concepto más general: "tránsito conmensurable" entre redes teóricas.

\footnotetext{
${ }^{6}$ Una primera versión estructuralista de la noción de incorporación se encontrará en Díez \& Moulines (2008, Cap. 13.4). La versión actual aparece como más adecuada y "elegante".

${ }^{7}$ No he demostrado formalmente este teorema, pero me parece plausible vistas las nociones en cuestión. La posibilidad de una prueba formal del teorema dependerá, en lo esencial, de la definición de reducción adoptada (pues la equivalencia y la aproximación son "variaciones" de la reducción). El problema estriba en que no existe todavía un consenso (ni entre estructuralistas ni entre no-estructuralistas) acerca de cuál es la elucidación formal más adecuada de la reducción interteórica. Ahora bien, si aceptamos que, sea cual sea la elucidación definitiva, un componente esencial de esta será lo que en Moulines (1984) denominé "reducción ontológica”, entonces parece que la prueba del teorema será bastante directa, dado que la reducción ontológica se puede definir justamente en términos de lo que aquí llamo "subestructuras parciales escalonadas".
} 
$N$ transita (conmensurablemente) hacia $N^{*}$ syss

(1) $N^{*} \pi N$

(2a) $N$ y $N^{*}$ forman parte de una misma evolución teórica

o bien

(2b) $N$ es incorporable en $N^{*}$

o bien

(2c) $N^{*}$ es incorporable en $N$

\section{Suplantación con inconmensurabilidad (parcial)}

Sean dos redes teóricas $N$ y $N^{*}$. Diremos que $N$ es suplantada (con inconmensurabilidad parcial) por $N^{*}$ syss:

(1) Ningún modelo actual en $N$ es subestructura parcial escalonada de algún modelo actual en $N^{*}$.

(2) (Casi todas) las aplicaciones intencionales exitosas en $N$ (o partes de las mismas) son subestructuras parciales escalonadas de aplicaciones intencionales en $N^{*}$.

(3) Existen aplicaciones intencionales no-exitosas en $N$ ("anomalías") que son subestructuras parciales escalonadas de aplicaciones intencionales exitosas en $N^{*}$.

En las suplantaciones se da el fenómeno de la "inconmensurabilidad" en el sentido de que en ellas constatamos la presencia de conceptos T-teóricos de $N$ que no son reproducibles mediante los conceptos T-teóricos de $N^{*}$ (ni siquiera como subestructuras parciales escalonadas), y por consiguiente tampoco son reproducibles en $N^{*}$ las leyes fundamentales de $N$. Sin embargo, en tales casos, la inconmensurabilidad solo puede ser parcial, pues las dos teorías deben ser comparables en cuanto a sus éxitos respectivos. Ello no significa que las aplicaciones intencionales de $N$ deban necesariamente reaparecer de forma idéntica entre las de $N^{*}$; pero al menos sus componentes, y por tanto las aplicaciones mismas, deben ser reconstruibles como subestructuras parciales escalonadas de las aplicaciones intencionales de $N^{*}$.

\footnotetext{
${ }^{8}$ Kuhn y Stegmüller han alegado en algunos escritos que hay casos históricos de "revoluciones científicas" (correspondientes a lo que aquí denominamos "suplantación”), en los que se "pierden" u "olvidan" algunas aplicaciones intencionales exitosas del paradigma precedente a la revolución que no tienen cabida en el paradigma posterior a la revolución. (Stegmüller llama a este supuesto fenómeno diacrónico "Kuhn-loss", o sea, "pérdida a lo Kuhn”.) Es una cuestión controvertida si existen ejemplos históricos reales que puedan plausiblemente interpretarse en este sentido. Pero, por si acaso, añadimos en nuestra condición (2) la cualificación "casi todas", pues, aun en el caso de que hubiera ejemplos históricos de "pérdidas a lo Kuhn" en un proceso de suplantación, tales pérdidas serán sin duda en número escaso por comparación con el excedente de éxitos de la teoría posterior: de lo contrario ningùn científico en su sano juicio estaría a la larga dispuesto a suplantar la primera teoría por la segunda.
} 


\section{Conclusión}

La argumentación llevada a cabo hasta aquí muestra que los cuatro tipos fundamentales examinados de desarrollo teórico en las ciencias empíricas (cristalización, evolución, incorporación y suplantación), para cada uno de los cuales se pueden aducir ejemplos históricos ya reconstruidos, son perfectamente "formalizables" de manera uniforme mediante el aparato usual de la metateoría estructuralista, enriquecido con la noción "conjuntista" general de subestructura parcial escalonada.

Quod erat demonstrandum

\section{Bibliografía}

Balzer, W., Moulines, C.U. y J.D. Sneed, (1987), An Architectonic for Science. The Structuralist Program, Dordrecht: Reidel.

Caamaño, M. (2009), “A Structural Analysis of the Phlogiston Case”, Erkenntnis 70(3): 331-364.

Clausius, R.J. (1850), "Über die bewegende Kraft der Wärme”, Annalen der Physik 79: 368-397, 500-524.

Clausius, R.J. (1854), "Über eine veränderte Form des zweiten Hauptsatzes der mechanischen Wärmetheorie", Annalen der Physik 93: 481-506.

Díez, J.A. y C.U. Moulines (2008), Fundamentos de Filosofía de la Ciencia, 3a edición revisada, Barcelona: Ariel.

Kuhn, T.S. (1962), The Structure of Scientific Revolutions, Chicago: The University of Chicago Press, 2a ed. , incluyendo "Postscript: 1969", 1970.

Kuhn, T.S. (1976), “Theory-Change as Structure-Change: Comments on the Sneed Formalism", Erkenntnis 10(2): 179-199.

Kuhn, T.S. (2000), The Road since Structure, Chicago: The University of Chicago Press.

Lakatos, I. (1970), "Falsificationism and the Methodology of Scientific Research Programmes", en Lakatos I. y A. Musgrave (eds.) (1970), Criticism and the Growth of Knowledge, Cambridge: Cambridge University Press.

Laudan, L. (1977), Progress and Its Problems, Berkeley: University of California Press.

Lorenzano, P. (2006), "La emergencia de un programa de investigación en genética”, en: Lorenzano, P., Martins L.A.-C.P. y A.C. Regner (eds.), Ciências da vida: estudos filosóficos e históricos, Filosofia e História da Ciência no Cone Sul, vol. 2, Campinas: Associação de Filosofia e História da Ciência do Cone Sul, pp. 333-360.

Lorenzano, P. (2008), "Inconmensurabilidad teórica y comparabilidad empírica", Análisis Filosófico 28(2): 239-279.

Ludwig, G. (1978), Die Grundstrukturen einer physikalischen Theorie, Berlin: Springer.

Manzano, M. (1989), Teoría de modelos, Madrid: Alianza.

Moulines, C.U. (1982), Exploraciones metacientificas, Madrid: Alianza. 
Moulines, C.U. (1984), “Ontological Reduction in the Natural Sciences”, en Balzer, W., Pearce, D. y H.-J. Schmidt (eds.), Reduction in Science, Dordrecht: Reidel, 1984, pp. 51-70.

Moulines, C.U. (1991) "Pragmatisch-diachronische Aspekte der Wissenschaftstheorie”, Untersuchungen zur Logik und zur Methodologie 8: 1-21.

Moulines, C.U. (1996), "Zur Typologie wissenschaftlicher Entwicklung nach strukturalistischer Deutung”, en Hubig, C. y H. Poser (eds.), Cognitio Humana - Dynamik des Wissens und der Werte, XVII, Leipzig: Institut für Philosophie der Universität Leipzig, pp. 397-410.

Moulines, C.U. (2010), “The Crystallization of Clausius's Phenomenological Thermodynamics”, en Ernst G. y A. Hüttemann (eds.), Time, Chance and Reduction, Cambridge: Cambridge University Press, pp. 139-158.

Scheibe, E. (1997), Die Reduktion physikalischer Theorien, Teil I, Grundlagen und elementare Theorie, Berlin: Springer

Sneed, J.D. (1978), The Logical Structure of Mathematical Physics, Dordrecht: Reidel, 2nd ed.

Stegmüller, W. (1973), Theorienstrukturen und Theoriendynamik, Berlin: Springer. 